\title{
NOTE ON A STIELTJES TYPE OF INVERSION
}

\author{
PASQUALE PORCELLI
}

If $F(z)$ is an analytic function for $z \notin[-\infty,-1], g(t)$ of bounded variation and real valued for $0 \leqslant t \leqslant 1$ and

$$
F(z)=\int_{0}^{1}(1+z t)^{-1} d g(t)
$$

then the Stieltjes type of inversion between $F(z)$ and $g(t)$ (cf. 1, p. 339, Theorem $7 \mathrm{a}$ ) is

$$
\lim _{y \rightarrow 0+} \frac{-1}{\pi} \int_{0}^{u} \frac{1}{t} I_{m} F\left(-\frac{1}{t}+i y\right) d t=\frac{g(u+)+g(u-)}{2}-\frac{g(v+)+g(v-)}{2},
$$

where $0 \leqslant v<u \leqslant 1, I_{m} F(z)$ is the imaginary part of $F(z)$ and $z=-t^{-1}+i y$.

A second type of inversion between $F(z)$ and $g(t)$ was obtained by Widder $(1$, p. 340, Theorem $7 \mathrm{~b})$ under the additional hypothesis that $g(t)$ is an absolutely continuous function. In the following theorem we shall establish an inversion between $F(z)$ and the right- and left-hand derivatives of $g(t)$ without the restriction that $g(t)$ be an integral.

Theorem. Let $F(z)$ be analytic for $z \notin[-\infty,-1], g(t)$ real valued and of bounded variation on $[0,1]$ and

$$
F(z)=\int_{0}^{1}(1+z t)^{-1} d g(t)
$$

then

$$
\lim _{y \rightarrow 0+} \frac{-1}{\pi t} I_{m} F\left(\frac{-1}{t}+i y\right)=\frac{g^{\prime+}(t)+g^{\prime-}(t)}{2}
$$

for any $t$ in $(0,1)$ at which the right- and left-hand derivatives $g^{\prime+}(t)$ and $g^{\prime-}(t)$ exist.

Proof. Let us suppose that $g(0)=0,0<t_{0}<1$ and that $g^{\prime+}\left(t_{0}\right)$ and $g^{\prime}-\left(t_{0}\right)$ exist. If we set

$$
R(t)=\left[\left(t_{0}-t\right)^{2}+\left(t_{0} y t\right)^{2}\right]^{-1}
$$

and $s=t_{0} \pi^{-1}$, then from (1) we have

$$
\frac{-1}{\pi t_{0}} I_{m} F\left(\frac{-1}{t}+i y\right)=s y \int_{0}^{t_{0}} t R(t) d g(t)+s y \int_{t_{0}}^{1} t R(t) d g(t) .
$$

Received December 20, 1955 and in revised form March 9, 1956. Work on this paper was sponsored by the Air Research and Development Command, under Contract AF 18 (600) 1393. 
In the first integral of this expression we can replace $g(t)$ by $\left[g\left(t_{0}\right)+g^{\prime}-\left(t_{0}\right)\right.$ $\left.\left(t-t_{0}\right)+h(t)\left(t-t_{0}\right)\right]$, where $h(t)$ is continuous at $t_{0}$ and $h\left(t_{0}\right)=0$, so that

$$
s y \int_{0}^{t_{0}} t R(t) d g(t)=s y g^{-}\left(t_{0}\right) \int_{0}^{t_{0}} t R(t) d t+s y \int_{0}^{t_{0}} t R(t) d h(t)\left(t-t_{0}\right) .
$$

The first term on the right side of this equation can be integrated directly and we can easily verify that it approaches $2^{-1} g^{\prime-}\left(t_{0}\right)$ as $y$ approaches $0+$. Upon using the integration by parts formula, the second term reduces to

$$
\text { - sy } \int_{0}^{t_{0}} h(t)\left(t-t_{0}\right)\left[R(t)+t R^{\prime}(t)\right] d t .
$$

If $J$ denotes the value of the last expression, then

$$
|J|<\frac{2 y}{\pi} \int_{\theta}^{t_{0}}|h(t)| R(t) d t
$$

For each $\epsilon>0$, there exists $\gamma>0$ such that $|h(t)|<\frac{1}{2} \epsilon t_{0}^{2}$ for $t_{0}-t<\gamma$, so that

$$
\frac{2 y}{\pi} \int_{t_{0}-\gamma}^{t_{0}}|h(t)| R(t) d t<\frac{2 \epsilon y t_{0}^{2}}{\pi} \int_{0}^{1} R(t) d t<\epsilon
$$

for $y>0$. Since $h(t)$ is a bounded function, there exists $\gamma^{\prime}>0$ such that, for $y<\gamma^{\prime}$,

$$
\frac{2 y}{\pi} \int_{0}^{t_{0}-\gamma}|h(t)| R(t) d t<\epsilon .
$$

In order to treat the second integral appearing in (2) we replace $g(t)$ by

$$
\left[g\left(t_{0}\right)+g^{\prime+}\left(t_{0}\right)\left(t-t_{0}\right)+k(t)\left(t-t_{0}\right)\right]
$$

and proceed as above. However, in this case the integration by parts formula yields the additional term

$$
\pi^{-1} y\left[k(1)\left(1-t_{0}\right) / t_{0}^{2}\left(1+y^{2}\right)\right]
$$

which approaches zero with $y$. This completes the proof of the Theorem.

Remark added in the revision. I am indebted to the referee for suggesting the revised form of the Theorem. Also, as he points out, the Theorem is valid if we replace the interval of integration $[0,1]$ by the ray $[0, \infty]$ and restrict $z$ so that $z \notin[-\infty, 0]$.

\section{REFERENCE}

1. D. V. Widder, The Laplace Transform, (Princeton, 1946).

Illinois Institute of Technology, Chicago 